\title{
Otimização multicritério para o problema de localização de centros de distribuição de uma empresa com unidade produtiva no Polo Industrial de Manaus
}

\section{Multi-criteria optimization for the location problem of distribution centers of a company with production plant in the Industrial Pole of Manaus}

\author{
Cauê Sauter Guazzelli' \\ Claudio Barbieri da Cunha ${ }^{1}$
}

\begin{abstract}
Resumo: Neste trabalho é apresentado um modelo matemático aplicado a um problema real de localização de centros de distribuição na Região Nordeste do Brasil para uma indústria que tem toda sua produção em uma região remota do Brasil. Nessa modelagem foram considerados critérios de decisão não aplicados conjuntamente na literatura científica. Mais especificamente é proposto um modelo de programação inteira mista que considera três camadas de instalações, dois tipos de modais, restrições de capacidade, custos fixos e variáveis para as unidades, além de múltiplos critérios, como custo operacional e de investimento, nível de serviço, impactos na sociedade e questões operacionais para a função de valor atribuída a cada solução. O modelo permite a determinação dos melhores locais para centros de distribuição e do modal utilizado para seu abastecimento, de forma a maximizar o valor da solução. Por meio da aplicação do modelo foram encontradas e comparadas soluções com altos valores de função objetivo. Os resultados foram obtidos considerando dados reais da empresa e possibilitaram a identificação de uma solução com valor 39\% maior em relação à solução com a estrutura logística atual.
\end{abstract}

Palavras-chave: Localização de instalações; Programação multicritério; Programação linear inteira.

\begin{abstract}
This paper presents a mathematical model applied to a real problem of location of distribution centers in the northeast region of Brazil for an industry that has all its production in a remote region of the country. The model considers decision criteria not jointly applied in the scientific literature. Specifically, we propose a mixed-integer linear programming model that considers three layers, two types of modals, capacity constraints, fixed and variable facility costs, and multiple criteria such as operating and investment costs, level of service, society impact, and operational issues for the value function assigned to each solution. The model allows the determination of the best locations for distribution centers and which modal should be used for its supply to maximize the value of the solution. The application of the model resulted in different solutions with high values of objective functions. The results were obtained using real data and a solution with 39\% value improvement compared with the current logistic structure was found.
\end{abstract}

Keywords: Facility location/location analysis; Multi-criteria decision; Integer linear programming.

\section{Introdução}

O objetivo deste trabalho é apresentar um modelo de programação inteira mista que considera múltiplos critérios na função objetivo a um problema real de localização de centros de distribuição na Região Nordeste do Brasil para uma indústria que tem toda sua produção em uma região remota do Brasil. $\mathrm{Na}$ modelagem foram considerados critérios de decisão não aplicados conjuntamente na literatura científica, como custo operacional e de investimento, nível de serviço, impactos na sociedade e questões operacionais.

É usual que grandes indústrias no Brasil tenham sua estrutura logística de forma que suas fábricas abasteçam os centros de distribuição e esses atendam os revendedores, que por sua vez atuam na venda e distribuição para o consumidor final, seguindo uma

\footnotetext{
${ }^{1}$ Departamento de Engenharia de Transportes, Escola Politécnica, Universidade de São Paulo - USP, São Paulo, SP, Brasil, e-mail: caue@inpo.eng.br; cbcunha@usp.br
}

Recebido em Jan. 14, 2015 - Aceito em Abr. 8, 2015

Suporte financeiro: Conselho Nacional de Desenvolvimento Científico e Tecnológico, CNPq. 
estrutura de rede hierárquica. No entanto, podem existir características e condicionantes práticas que influenciam as tomadas de decisão estratégicas e que podem tornar sua modelagem matemática mais complexa.

Dentro dessas características se enquadra a utilização de outros aspectos além do custo para auxílio na tomada de decisões estratégicas como a decisão de localização de centros de distribuição. Ainda que normalmente o fator primordial considerado nesse tipo de decisão seja o custo total (o de investimento e o de operação), algumas soluções alternativas com custos muito próximos ao custo da solução ótima podem se mostrar viáveis se se destacarem em outros aspectos normalmente não considerados na modelagem.

Um desses aspectos são os diferentes acordos de nível de serviço firmados com os revendedores. Em função do ambiente competitivo atual, a redução do lead time entre pedido e entrega e aumento de frequência de entrega podem se tornar questões preponderantes na decisão da localização de centros de distribuição, pois podem funcionar como diferenciais comerciais para a empresa.

Outro aspecto que também pode ser levado em consideração é o impacto social na imagem da empresa que as tomadas de decisões causam em relação à sociedade. $\mathrm{O}$ fechamento e a abertura de unidades são exemplos de medidas que provocam repercussão na sociedade, sobretudo no que tange à criação ou eliminação de vagas de trabalho. Aspectos ambientais também podem fortalecer a imagem da empresa, como tomadas de decisão que proporcionam a redução da emissão de gases poluentes, como o $\mathrm{CO}_{2}$.

Além disso, a complexidade da operação, retratada pelo excesso de unidades logísticas, associada à dificuldade de gestão que isso pode causar também podem ser levadas em consideração durante a tomada de decisão da localização de centros de distribuição.

Apesar do vasto material bibliográfico existente sobre a utilização de modelos de programação inteira mista com o objetivo de encontrar uma configuração de rede cujo custo operacional decorrente seja mínimo, esses fatores apontados, também importantes na tomada de decisão, muitas vezes são tratados exogenamente aos modelos de otimização.

Este trabalho propõe a aplicação de uma análise multicritério de função de valor e sua inserção em um modelo de otimização, a fim de possibilitar a geração de cenários alternativos cujos valores podem ser maiores que o valor da solução de menor custo operacional.

Para tal, uma ferramenta em planilha eletrônica foi desenvolvida, na qual foi construído um modelo discreto de localização de centros de distribuição capacitados, com três camadas de unidades logísticas (fábrica, portos e centros de distribuição), e que considera a cadeia logística desde a fábrica até a distribuição final aos clientes (para esse caso, empresas revendedoras são consideradas os clientes da empresa produtora).

Funções de valor que representam os objetivos fundamentais para a decisão foram inseridas em sua função objetivo, essas funções foram classificadas em quatro grupos: Custo, Nível de Serviço, Impacto na Sociedade e Impacto na Operação.

Uma vez que os modelos encontrados na literatura científica não consideram em uma única modelagem todos esses aspectos em sua função objetivo, é proposta neste trabalho uma formulação baseada em programação inteira mista que possibilita considerá-los com a finalidade de atender a necessidade de representação dos elementos considerados na tomada de decisão por parte de empresas de grande porte no Brasil.

Consequentemente, o modelo proposto, que tem por finalidade a localização de centros de distribuição capacitados, considerando custos variáveis, tem como principais características ser um modelo multicritério, com três camadas de unidades logísticas, hierárquico, com restrição de volume mínimo necessário para abertura de CD ou utilização de porto, além de outras características que serão detalhadas adiante.

O restante deste artigo está organizado da seguinte forma: a seção 2 contempla uma revisão bibliográfica a fim de situar o trabalho no contexto da pesquisa em localização. A seção 3 apresenta uma breve descrição da metodologia utilizada no estudo, enquanto a seção 4 apresenta a caracterização do problema para o qual o modelo matemático foi aplicado. A seção 5 apresenta o detalhamento e formulação do modelo matemático. A seção 6 mostra a aplicação do modelo ao caso real e os resultados das modelagens. A seção 7 apresenta as considerações finais.

\section{Revisão bibliográfica}

Segundo Current et al. (2002), não só o problema de localização é inerente a diversos setores da sociedade como as decisões de localização são frequentemente feitas em todos os níveis das organizações humanas, desde indivíduos e famílias, até empresas, agências governamentais e agências internacionais.

O tipo de modelagem matemática mais usual trata do problema de localização discreta, que consiste na definição de, dado um conjunto de candidatos pré-selecionados, quais locais deverão ser selecionados para serem instaladas unidades logísticas. Daskin (1995) relaciona os principais tipos de modelos matemáticos discretos de localização já desenvolvidos. Esses modelos servem como base para as formulações derivadas que abordam outras características das operações logísticas. Brandeau \& Chiu (1989) apresentam uma resenha contemplando 54 trabalhos sobre localização com o objetivo de fornecer uma visão abrangente da maioria dos problemas estudados na época, com suas formulações, 
e como eles se relacionam entre si. Já Current et al. (2002) relacionam 51 referências bibliográficas sobre o assunto, envolvendo inúmeras aplicações diferentes, destacando seu aspecto multidisciplinar.

O chamado Modelo de Cobertura tem o objetivo de identificar o conjunto de instalações que garantam que todos os pontos de demanda tenham a distância da instalação mais próxima menor ou igual a uma distância definida D. Pode ser interpretado, no contexto logístico, como um modelo que busca uma solução com a menor quantidade de instalações que garanta um "nível de serviço" determinado. Nesse modelo, porém, o custo fixo não é considerado senão indiretamente por meio da busca da quantidade mínima de unidades necessárias.

Uma alternativa ao Modelo de Cobertura é o modelo de P-centros, que considera um número de instalações dado exogenamente. Seu objetivo então é a busca de uma configuração que minimize a distância máxima entre um ponto de demanda e a instalação mais próxima. Pode ser representado, no contexto logístico, como o objetivo de minimizar o pior nível de serviço dada uma quantidade de instalações já estipulada.

Já o Modelo de P-medianas é uma evolução do Modelo de P-centros e tem como função objetivo a minimização da distância total ponderada entre as instalações e os pontos de demanda a elas alocados.

Esse tipo de modelo desempenha uma melhor aproximação ao objetivo de minimizar o custo operacional melhorando o nível de serviço médio, dada uma quantidade de instalações fixa. Essa modelagem pode ser interpretada como se os custos fixos de todos os candidatos fossem iguais. Se a informação de distância entre os candidatos a instalações e os clientes for interpretada como uma aproximação do custo de transporte por unidade de volume, o modelo se aproximará ainda mais de uma operação real. Para mais detalhes, recomendam-se os trabalhos de Daskin (1995), Drezner (2004) e ReVelle \& Eiselt (2005). Exemplos de aplicação desse tipo de modelo podem ser vistos em Amaral et al. (2012) e Mapa \& Lima (2012).

Algumas hipóteses do problema das P-medianas podem não ser apropriadas para algumas situações que envolvem a localização de instalações, em particular: (i) todas as instalações têm o mesmo custo fixo; (ii) as instalações não têm limite de capacidade; (iii) o número de instalações a serem selecionadas é definido a priori (igual a p).

Uma generalização do problema das P-medianas consiste na consideração dos custos fixos de cada instalação candidata a ser aberta, o que define o chamado Problema de Localização de Planta Simples (Simple Plant Location Problem). Essa característica implica que o modelo consegue definir também qual é a quantidade ideal de instalações a serem abertas, de tal forma que o custo total, composto das parcelas custo de transporte e custo fixo, seja minimizado. Boas referências sobre esse problema podem ser encontradas em Mirchandani \& Francis (1990) e ReVelle et al. (2008).

O Problema de Localização de Instalações Capacitadas (Capacitated Facility Location Problem) corresponde à generalização do Problema de Localização de Planta Simples em que são conhecidas as capacidades de cada candidato. Essa restrição impõe soluções em que alguns clientes podem não ser atendidos pela instalação selecionada mais próxima. Para boas referências podem-se citar Sridharan (1995), Aardal et al. (2015) e Rahmaniani \& Ghaderi (2015).

Outro aspecto que também pode ser considerado a fim de retratar problemas reais é o ganho de escala nos custos dos centros de distribuição, como fazem Guazzelli \& Cunha (2014) em uma abordagem que define diferentes grupos de CDs baseada em seu tamanho e permite que o modelo de otimização escolha qual é o melhor tamanho para cada local.

Algumas outras restrições e condicionantes podem ser contemplados em modelos mais complexos voltados a resolver problemas reais encontrados na prática. Esses são o caso de restrições que garantem que o tempo de deslocamento ou a distância dos clientes até o ponto definido para seu atendimento seja menor ou igual aos acordos de nível de serviço estipulados, como em Lee \& Dong (2008), Pasandideh et al. (2014) e Farahani et al. (2014), e da definição de faixas de número de unidades permitidas para cada camada da cadeia, como em Altiparmak et al. (2006), Hinojosa et al. (2008) e Pati et al. (2008).

As características citadas acima, além de geralmente serem de fácil utilização nos modelos, restringem o espaço de soluções possíveis, o que pode inclusive acarretar na redução do tempo computacional de resolução. Esse também é o caso da imposição de abertura de certa quantidade de unidades em determinadas regiões, decorrente de decisões comerciais, porém esse aspecto não tem sido explorado na literatura.

Partindo dos modelos de localização clássicos, uma série de outros aspectos e condicionantes pode ser incorporada isoladamente ou em conjunto. A atual literatura científica vem desenvolvendo alguns modelos que apresentam combinações de características, permitindo a modelagem cada vez mais fidedigna de problemas reais.

Melo et al. (2009) fazem uma vasta revisão de modelos de localização no contexto da cadeia de suprimento, na qual apontaram aspectos que caracterizam os modelos encontrados na literatura, entre eles quantidade de camadas, de tipos de produtos e de períodos, se os parâmetros são determinísticos ou estocásticos, capacidade de instalações, custo de inventários, custos de produção, restrições de orçamento, além de outras ainda não tão abordadas 
pelos trabalhos científicos e que permitem traduzir de melhor forma a peculiaridade das operações reais, apresentando uma taxonomia e classificando os modelos recentes.

Em função de o problema de localização e alocação se enquadrar no grupo de problemas estratégicos, apresentando uma grande quantidade de agentes envolvidos com diferentes interesses, com múltiplos objetivos conflitantes, questões fundamentais intangíveis e requerendo soluções robustas que apresentem bom desempenho em diversas ocasiões, a abordagem da análise multicritério é adequada.

A utilização de técnicas de análises multicritério associadas a modelos de otimização vem sendo bastante estudada, sendo uma área com grande potencial de desenvolvimento, uma vez que considera aspectos importantes de problemas reais diversos e possibilita a geração de soluções alternativas às que minimizam somente o custo operacional, como é o caso de Passos \& Souza (2013), que desenvolveram um método multicritério com base em Análise Hierárquica (AHP) aplicado à comparação de distribuidoras de energia elétrica.

Farahani et al. (2010) fizeram uma extensa revisão sobre problemas de localização multicritério, classificando-os em problemas de decisão de múltiplos atributos (MADM - Multiple Attribute Decision Making), nos quais existe uma quantidade limitada de alternativas predeterminadas e problemas de decisão de múltiplo objetivo (MODM - Multiple Objective Decision Making), cujas técnicas buscam a definição de uma melhor alternativa que satisfaça o tomador de decisão, de forma a atingir níveis aceitáveis de desempenho a um conjunto de objetivos. Ainda no mesmo trabalho levantam alguns critérios considerados nos objetivos dos problemas do tipo MODM, que foram classificados em critérios de custo, de riscos ambientais, de cobertura de atendimento, de nível de serviço, de lucro e de outros tipos.

No trabalho de Melo et al. (2009) são apresentados alguns artigos que aplicam modelos multicritério ao problema de planejamento de redes de supply chain, como é o caso de Guillén et al. (2005), que consideram na função objetivo não apenas o lucro mas também o atendimento da demanda em um modelo de localização de fábricas e centros de distribuição capacitados, que considera aspectos realistas, sendo multiproduto, estocástico e com duas camadas. Já Altiparmak et al. (2006) utilizam um algoritmo genético desenvolvido para propor soluções de boa qualidade, de forma a possibilitar sua comparação e escolha por parte do tomador de decisão. Farahani \& Asgari (2007) combinam aspectos de decisão multicritério com técnicas de cobertura de forma a encontrar soluções que impliquem em um baixo custo fixo associado à menor quantidade de CDs possíveis, e cujas localidades apresentem maior função de valor possível, associada a critérios levados em consideração pelos tomadores de decisão.

Recentemente muitos estudos têm focado no desenvolvimento de algoritmos e heurísticas para o problema de planejamento de redes de supply chain com estrutura hierárquica e um produto. Latha Shankar et al. (2013) aplicaram um algoritmo evolucionário ao problema, com função objetivo considerando quantidade de plantas, fluxo de materia-prima, quantidade de produtos entre plantas e CDs e entre CDs e clientes, além do custo logístico da cadeia. Olivares-Benitez et al. (2013) desenvolveram um modelo linear inteiro (aplicado a pequenas instâncias) e uma meta-heurística que combina princípios de funções gulosas, scatter search, path relinking e programação linear, levando em conta objetivos como custo logístico e tempo de transporte máximo entre CD e cliente. Ozgen \& Gulsun (2014) combinam a utilização de programação linear com técnicas de AHP para propor soluções que consideram tanto aspectos quantitativos quanto qualitativos. Harris et al. (2014) desenvolveram um algoritmo evolucionário que particiona o problema em fases, sendo elas a de localização dos CDs e de alocação dos clientes, de forma a obter soluções com baixo custo e baixa emissão de $\mathrm{CO}_{2}$.

Com uma aplicação a um caso no Brasil, Montibeller \& Yoshizaki (2011) propõem uma estrutura de análise de decisão multicritério para problemas de localização de instalações logísticas, sugerindo uma forma alternativa de análise que combina o valor da topologia da rede com valores dos nós escolhidos para chegar a um valor global da cadeia de suprimentos.

Os modelos a serem apresentados na seção 5 avançam na literatura descrita acima ao apresentar um modelo de programação linear inteira mista aplicado a um problema mais abrangente no contexto de problemas de decisão de múltiplo objetivo (MODM) de localização de centros de distribuição. Isso significa que os aspectos considerados nas tomadas de decisão reais, não considerados em um único modelo até então na literatura científica, foram considerados explicitamente no modelo apresentado, sendo eles custo operacional, custo de investimento, nível de serviço dado em termos de lead time para dois grupos de clientes, bem como frequência de entregas, impactos das decisões na sociedade, sendo eles emissão de $\mathrm{CO}_{2}$, quantidade de $\mathrm{CDs}$ abertos e fechados e impacto na operação retratado pela quantidade de centros de distribuição, além de considerar três camadas de instalações capacitadas e dois tipos de modais.

\section{Metodologia utilizada}

A metodologia utilizada no estudo é apresentada na Figura 1 e foi formada por elementos de análises multicritério de funções de valor e de estudos de 


\section{Estruturação \\ do problema \\ de decisão

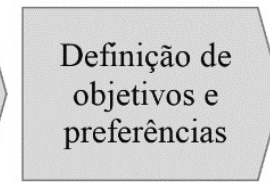

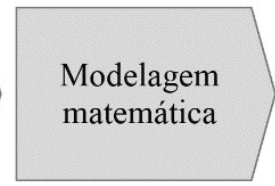

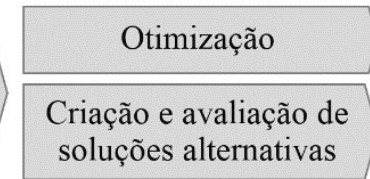

Análise dos

resultados

obtidos

Figura 1. Metodologia aplicada ao estudo.

otimização com modelagem de programação inteira mista.

Uma vez estruturado o problema e definidos os objetivos, um modelo multicritério de análise de decisão foi desenvolvido, a partir do qual o modelo de programação inteira mista multicritério foi construído. O problema foi abordado dessa forma em função de sua complexidade, que permite uma quantidade combinatória de soluções.

Por meio do modelo de otimização foi possível obter a configuração logística de maior valor em termos dos objetivos previamente definidos. Para permitir a geração de soluções alternativas que pudessem ser comparadas à solução de maior valor, alguns cenários foram rodados conforme parâmetros foram alterados no modelo, como será detalhado na seção de aplicação.

\section{Caracterização do problema}

$\mathrm{O}$ desenvolvimento desta pesquisa considera o planejamento de redes com definição da localização de centros de distribuição e fluxos de transporte para uma indústria que tem sua planta de produção localizada na Zona Franca de Manaus e que atua na Região NE do Brasil. Outros estudos já vêm abordando o planejamento de redes com fábricas localizadas em Manaus, em função das peculiaridades desses problemas, como é o caso de Medeiros et al. (2015).

Esta seção apresentará aspectos peculiares à operação atual da indústria que motivaram o desenvolvimento do modelo matemático e sua aplicação.

a. Contextualização da Zona Franca de Manaus

Por meio da criação da Zona Franca de Manaus, modelo de desenvolvimento econômico implantado pelo governo brasileiro para promover o desenvolvimento econômico da Região Amazônica Ocidental, existem incentivos fiscais significativos para a região, com redução e até isenção de alguns impostos.

A Região Amazônica, onde Manaus está localizada, abriga a maior floresta tropical do mundo, que impõe grande dificuldade de deslocamento. O principal meio de abastecimento da região é o modal hidroviário fluvial, que conecta a cidade a outras capitais do país, como Belém e Porto Velho, e ao Oceano Atlântico, por onde é possível completar o percurso de cabotagem a portos marítimos na costa brasileira. Em função dessas peculiaridades geográficas, o transporte na região se torna difícil e lento, o que implica em custos que se comportam de forma mais complexa em relação às variáveis de transporte, especialmente distância e velocidade.

b. Características da operação atual

A empresa tem uma operação que cobre grande parte da cadeia logística, incluindo produção, abastecimento dos CDs, armazenagem e distribuição até os pontos de venda (revendedores).

A empresa possui apenas uma fábrica, localizada em Manaus, um centro de distribuição na cidade de Belém e quatro centros de distribuição ao longo da Região Nordeste do Brasil e que foram localizados ao longo do desenvolvimento da empresa, sem utilização de estudo de otimização.

Em função da geografia da Região Amazônica, o transporte de transferência até o centro de distribuição é iniciado por transporte hidroviário, que carrega os produtos dentro de carretas ou contêineres até os portos fluviais ou marítimos. Entre os portos e os centros de distribuição, as carretas ou contêineres são acoplados aos cavalos mecânicos, que efetuam a segunda parte da transferência por transporte rodoviário.

Os centros de distribuição não mantêm estoque, a não ser o estoque dos produtos que estão em processo de transbordo durante a operação de cross dock que se realiza nos CDs.

c. Estruturação do problema de decisão

O problema de decisão foi estruturado com base nos objetivos da empresa, que eram redução do custo na operação de distribuição e melhoria do nível de serviço.

Para o estudo, foi definido que os tipos de decisão passíveis de serem realizadas para a construção de cada alternativa eram:

- Localização de pontos de distribuição (podendo-se manter ou não as unidades atuais);

- Quantidade de produtos de abastecimento que cada CD recebe vindo de cada porto;

- Quantidade de produtos enviados a cada revendedor partindo de cada centro de distribuição.

Os valores de cada uma dessas variáveis apresentaram influência nos objetivos e preferências que representavam os valores da empresa.

Também foram levantados requisitos da operação que caracterizaram limites a partir dos quais as soluções se tornavam inaceitáveis para a empresa. Esses requisitos foram traduzidos em restrições no modelo de programação inteira mista. 


\section{Modelagem multicritério}

Nesta seção é apresentado o modelo matemático proposto para o problema de localização de centros de distribuição que incorpora as principais restrições e condicionantes encontradas na operação descrita acima.

A modelagem matemática foi desenvolvida em três etapas. Primeiramente, um modelo multicritério foi desenvolvido, com a definição de objetivos que representam as preferências da empresa e a construção da função de valor que os representa. Posteriormente, um modelo de programação inteira mista foi desenvolvido em planilha eletrônica para encontrar soluções de mínimo custo operacional. Por fim, ao modelo de otimização foram adicionados os elementos desenvolvidos na análise de decisão, chegando-se a um novo modelo de otimização que considerou múltiplos critérios em sua função objetivo.

a. Definição de objetivos e preferências

Por meio de discussões com os principais agentes envolvidos no processo, utilizando técnicas de listas de desejo e desenvolvendo alternativas muito boas e muito ruins, operacionalmente, a árvore de valor que representa a estrutura de objetivos fundamentais e apresentada na Figura 2 foi desenvolvida. Para detalhes em estruturação de problemas para modelagem multicritério, ver Franco \& Montibeller (2010).

Após a definição dos nove atributos utilizados para representar o valor da equipe em relação ao desempenho logístico de operação, os limites mínimos e máximos aceitáveis e possíveis, bem como uma função de valor para cada um deles, foram construídos.

Foi possível notar que alguns dos objetivos eram conflitantes, o que caracteriza a adequação desse tipo de análise ao problema. Também foi verificado que eles obtinham características desejáveis a atributos de uma análise multicritério, apontadas por Keeney \& Gregory (2005), como não redundância, serem preferencialmente independentes e operacionalmente mensuráveis, entre outros.

As análises se limitaram à modelagem de funções de valor, não levando em consideração aspectos de riscos e incertezas na análise. Todos os modelos foram carregados com dados determinísticos, estimados a partir das análises de dados desenvolvidas.

\section{i. Custo}

O custo operacional é caracterizado pelo custo mensal que é gasto na cadeia de distribuição da empresa. Nele estão contidos todos os custos de transporte, operação de unidades e taxas e impostos relativos a essa etapa da cadeia. Como os custos fixos da unidade oneram a operação dos distribuidores, porém não são cobrados diretamente da indústria, o modelo trata destes custos considerando-os "diluídos" no custo de distribuição.

Para delimitar os limites desse atributo, o custo inerente à rede atualmente utilizada foi considerado como o máximo aceitável. Já para custo mínimo possível foi considerado o custo inerente à solução ótima do modelo de minimização de custos operacionais que será apresentado na seção 5.b.i.

A função de valor desse atributo tem um comportamento linear, demonstrando que a redução de uma mesma quantidade de custo tem o mesmo valor, independentemente da faixa em que o custo operacional se encontra, desde que entre os valores limites definidos.

O investimento para alteração de configuração da rede de distribuição está associado à abertura e fechamento de centros de distribuição decorrentes de reformas nos novos locais e esforços de contratação para abertura e multas contratuais e trabalhistas para fechamento destas unidades.

\section{ii. Nível de serviço}

Os atributos de nível de serviço foram identificados como objetivos fundamentais que representam o valor da companhia. Eles podem representar vantagem competitiva para os revendedores, podendo ser fator decisivo de compra por parte dos consumidores.

O nível de serviço retrata a qualidade do serviço logístico prestado aos revendedores e foi medido por meio de dois indicadores principais, sendo eles o tempo médio de viagem até os revendedores e a frequência média de entregas na região.

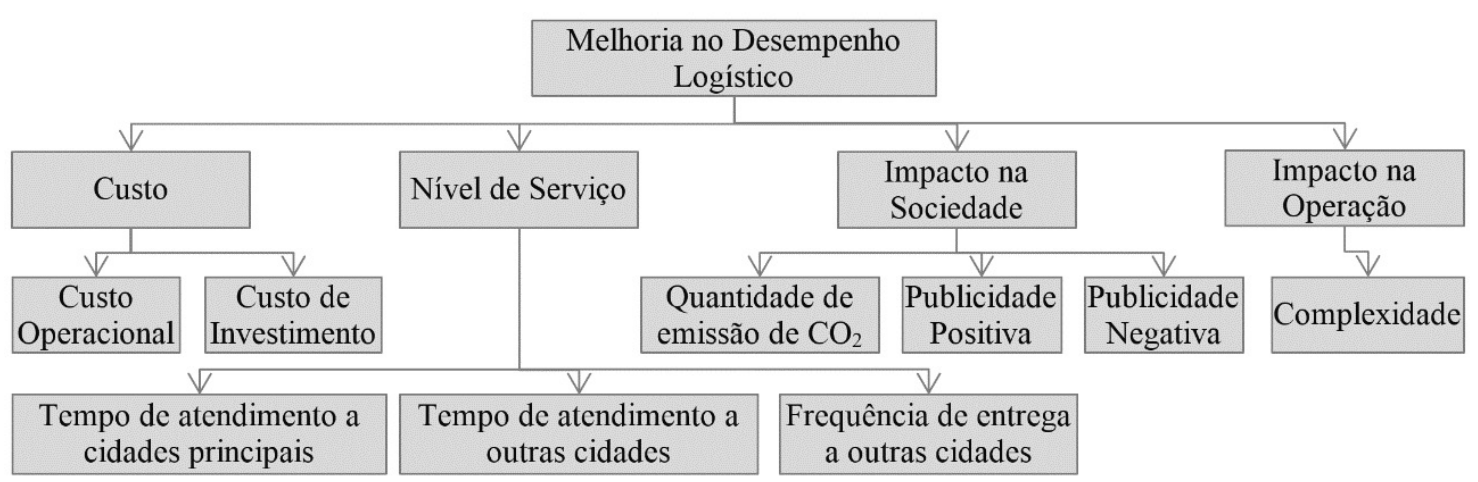

Figura 2. Árvore de valor. 
O valor do nível de serviço requerido para cada região pode ser diferente em função das diferentes características de cada mercado. Por isso, as cidades foram separadas em dois grupos, sendo o primeiro composto pelas cidades principais e o segundo pelas demais cidades.

Para o grupo das cidades principais foi definida a necessidade de haver uma frequência de atendimento diária e por isso esse atributo não foi considerado na função de valor, sendo descartada qualquer solução que não atendesse a esse requisito.

\section{iii. Impacto na sociedade}

A empresa entende que sua imagem perante a sociedade é um fator importante que deve ser levado em consideração em suas decisões estratégicas, sobretudo porque essa imagem se relaciona com o fortalecimento das marcas, que se reflete no desempenho de vendas.

O planejamento da malha logística de uma empresa de grande porte apresenta grande impacto na sociedade, para o qual foram definidos três atributos fundamentais, sendo eles publicidade positiva, publicidade negativa e emissão de $\mathrm{CO}_{2}$. Seu consumo foi estimado com base em taxas médias de emissão de $\mathrm{CO}_{2}$ por quilômetro de veículos novos.

A publicidade negativa foi representada no modelo pela quantidade de unidades logísticas que são fechadas. Sobretudo regionalmente, a visão que o consumidor tem ao saber do fechamento de uma unidade na região, que pode causar demissões e problemas na economia regional, pode ter impacto negativo na imagem da marca e nas vendas. De forma análoga, a abertura de uma nova unidade pode gerar um fortalecimento da marca na região em função da criação de novas vagas de emprego e promoção do desenvolvimento econômico.

A quantidade de $\mathrm{CO}_{2}$ emitida com a operação logística também foi considerada como atributo fundamental para a analise de decisão. Esse fator foi considerado em função tanto da necessidade de preservação do meio ambiente quanto da imagem que a empresa transmite ao consumidor quando considera esse aspecto em suas tomadas de decisão.

iv. Impacto na operação

A existência de grande quantidade de unidades logísticas pode apresentar um fator negativo em relação à operação, uma vez que implica na necessidade da gestão de equipes maiores, maior quantidade de fornecedores e contratos. Esse fator pode gerar uma complexidade para a operação e foi inserido no modelo, sendo representado pela quantidade de centros de distribuição ativos.

\section{v. Ponderação de pesos}

Uma vez definidos os limites aceitáveis ou possíveis e as funções de valor para cada um dos elementos que retratam o desempenho logístico, os pesos relativos foram definidos, por meio do método swing weight (Goodwin \& Wright, 2009). Os valores obtidos podem ser vistos na Tabela 1 .

\section{b. Modelos de otimização}

O modelo de minimização de custos operacionais considera as seguintes características: três camadas de instalações hierárquicas, capacidade máxima e volume mínimo do total movimentado por centro de distribuição, capacidade máxima e volume mínimo do total movimentado por porto, CDs com custos variáveis, limites máximo e mínimo de quantidade de CDs abertos, possibilidade de forçar a existência de CDs em determinadas locais.

O modelo de maximização da função de valor definida pela análise multicritério acrescenta ao modelo inicial restrições que retratam os limites aceitáveis e possíveis de cada um dos elementos que compõem a função de valor, sendo eles: custo máximo de operação, custo máximo de investimento, limite de lead time médio de clientes de cada grupo (principais e não principais) e de frequência de atendimento médio para clientes não principais, limites de quantidade de CDs abertos, limites de quantidade de CDs fechados e limite de emissão de $\mathrm{CO}_{2}$.

$\mathrm{O}$ modelo tem a finalidade de determinar a localização dos centros de distribuição $c$ e quais clientes $l$ devem ser atendidos por ele. Além disso, também determina por qual porto $p$ cada centro de distribuição $c$ é abastecido.

Trata-se de um modelo discreto, o que implica que o conjunto dos locais candidatos a abrigar CDs e o conjunto dos portos que podem ser utilizados devem ser definidos a priori. Os pontos de demanda e de eventuais unidades existentes (fábrica e CDs atuais) também são conhecidos a priori.

Em função de ser um modelo matemático do tipo discreto, é necessário conhecer as matrizes de custo de transporte entre fábrica, CDs e clientes; mais especificamente, os custos unitários de transferência $c p_{p}$ associados ao transporte entre a fábrica e os portos $p$ e $c p c_{p c}$ associados ao transporte entre os portos $p$ e os candidatos aos CDs $c$ e os custos unitários de distribuição $c c l_{c l}$ associados à distribuição entre os candidatos a $\mathrm{CD} c$ e os clientes $l$.

Tabela 1. Pesos de cada atributo $\left(p^{v}\right)$.

\begin{tabular}{ccccccccc}
\hline Custo & \multicolumn{3}{c}{ Nível de serviço } & \multicolumn{3}{c}{ Impacto na sociedade } & \multicolumn{2}{c}{ Impacto na operação } \\
\hline Custo & Investimento & $\Delta \mathrm{T}$ de & $\Delta \mathrm{T}$ de & Frequência & Emissão & Publicidade & Publicidade & Complexidade \\
operacional & & att. $\mathrm{C} 1$ & att. C2 & entrega C2 & de $\mathrm{CO}_{2}$ & positiva & negativa & \\
100 & 15 & 75 & 35 & 10 & 8 & 10 & 15 & 20 \\
\hline
\end{tabular}


O modelo considera fluxos da fábrica para os portos $p$ e entre os portos $p$ e os $\mathrm{CDs} c$. A partir dos CDs, são considerados fluxos que representam a distribuição até os clientes $l$. O modelo considera um único período e todos os parâmetros que representam volumes, como a demanda de cada cliente $d_{l}$ as capacidades, devem ser dados em relação ao período considerado, usualmente um ano ou um mês típico.

Para a utilização de um porto é necessário que o volume nele movimentado seja maior ou igual ao volume mínimo VminPorto e menor ou igual ao volume máximo VmaxPorto, dados em quantidade total de produtos movimentados no período. $\mathrm{O}$ mesmo ocorre com os candidatos a CDs, que devem movimentar um volume maior ou igual ao volume mínimo definido $V \min C D$ e menor ou igual à sua capacidade $V \max C D$. Além disso, o modelo considera para os centros de distribuição custos variáveis. O modelo também permite que a abertura de um determinado CD seja forçada.

i. Modelo de minimização de custos

Conjuntos:

C: conjunto de locais candidatos a CDs;

L: conjunto de clientes;

P: conjunto de portos possíveis.

Parâmetros:

CDmin: quantidade mínima de CDs ativos;

CDmax: quantidade máxima de CDs ativos;

$d_{\text {: }}$ demanda do cliente 1 ;

$V \min C D$ : volume mínimo para abertura de um $\mathrm{CD}$;

VmaxCD: capacidade máxima de movimentação por $\mathrm{CD}$;

VminPorto: volume mínimo para ativação de um porto;

VmaxPorto: capacidade máxima de movimentação por porto;

$f_{c_{c}}$ : valor binário que tem valor 1 se a abertura do CD c será forçada;

$c p_{p}$ : custo unitário de transporte marítimo entre a fábrica e o porto $\mathrm{p}$;

$c p c_{p c}$ : custo unitário de transferência entre o porto $\mathrm{p}$ e o $\mathrm{CD} \mathrm{c}$;

$\mathrm{ccl}_{c l}$ : custo unitário de distribuição desde CD c até o cliente 1;

$c v_{c}$ : custo variável unitário de movimentação de produto no $\mathrm{CD}$ c;
M: um número suficientemente grande.

Variáveis de decisão:

$z_{c}\left\{\begin{array}{l}1 \text { seoCDcestáativo } \\ 0 \text { casocontrário }\end{array} \quad\right.$, comc $\in \boldsymbol{C} ;$

$z_{p}\left\{\begin{array}{l}1 \text { seo porto pestáativo } \\ 0 \text { casocontrário }\end{array} \quad\right.$, com $p \in P$

$q p c_{p c}$ : quantidade de produto transportado entre o porto p e o CD c, $\operatorname{com} p \in \boldsymbol{P}$ e $c \in \boldsymbol{C}$

$q q l_{c l}$ : quantidade de produto transportado entre o CD c e o cliente $1, \operatorname{com} c \in \boldsymbol{C}$ e $l \in \boldsymbol{L}$

Restrições:

$$
\begin{gathered}
\sum_{p \in P} q p c_{p c} \geq \operatorname{VminCD} \cdot z_{c}, c \in \boldsymbol{C} \\
\sum_{c \in C} q p c_{p c} \geq \operatorname{VminPorto} \cdot z_{p}, p \in \boldsymbol{P} \\
\sum_{p \in P} q p c_{p c} \leq \operatorname{VmaxCD} \cdot z_{c}, c \in \boldsymbol{C} \\
\sum_{c \in C} q p c_{p c} \leq \operatorname{VmaxPorto} \cdot z_{p}, p \in \boldsymbol{P} \\
z_{c} \geq f c_{c}, c \in \boldsymbol{C} \\
\sum_{c \in C} q c l_{c l}=d_{l}, l \in \boldsymbol{L} \\
\sum_{p \in P} q p c_{p c}=\sum_{l \in L} q c l_{c l}, c \in \boldsymbol{C} \\
\sum_{c \in C} z_{c} \geq C D M i n \\
\sum_{c \in C} z_{c} \leq C D M a x
\end{gathered}
$$

As Restrições 1 garantem que se um CD for aberto, o volume que passará por ele deverá ser maior ou igual ao volume mínimo para abertura de um $\mathrm{CD}$, enquanto as Restrições 2 têm o mesmo efeito na ativação dos portos. As Restrições 3 restringem o volume máximo para CDs, além de garantir que apenas CDs ativos serão utilizados, enquanto as Restrições 4 restringem o volume máximo para os portos ativos e garante que apenas os portos ativos sejam utilizados. As Restrições 5 garantem a abertura de CDs se eles forem forçado. As Restrições 6 garantem o atendimento de toda a demanda aos clientes. As Restrições 7 garantem a continuidade de volume nos CDs, sendo que todo o volume recebido pelos portos é enviado a clientes. As Restrições 8 e 9 restringem a quantidade de CDs ativos.

Função objetivo:

Minimizar:

$$
\sum_{c \in \boldsymbol{C}} \sum_{p \in \boldsymbol{P}} q p c_{p c} \cdot\left(c p_{p}+c p c_{p c}+c v_{c}\right)+\sum_{c \in \boldsymbol{C} l \in \boldsymbol{L}} \sum_{c l} q l_{c l} \cdot c c l_{c l}
$$


A Função 10 é composta por dois elementos, sendo que o primeiro representa os custos de transporte entre a fábrica e os portos, entre os portos e os CDs e os custos variáveis dos $\mathrm{CDs}$, enquanto o segundo componente representa os custos de distribuição entre os CDs e os revendedores.

ii. Modelo de maximização da função de valor multicritério

Para inserir o modelo multicritério no modelo de otimização foram construídas funções lineares por partes, que representam a função de valor de cada atributo. A função objetivo do modelo original foi acrescida à soma dos valores de cada uma dessas funções lineares por partes para compor a função objetivo do novo modelo.

Dados o novo conjunto:

V: conjunto de objetivos fundamentais da função de valor da solução acrescidos ao custo operacional;

Considerando a inclusão no modelo dos parâmetros: $e c_{c}$ : cujo valor é igual a 1 se o $\mathrm{CD} c$ está ativo atualmente e 0 caso contrário;

$c a$ : custo de abrir um novo $\mathrm{CD}$;

$c f$ : custo de fechar um CD atualmente aberto;

$b_{j}^{V}$ : ponto de quebra $j$ na função de valor do atributo $v, \operatorname{com} j=1,2, \ldots, n+1$ e $v \in \mathrm{V}$;

$g_{l}$ : cujo valor é igual a 1 se o cliente $l$ está em uma cidade pertencente ao grupo das cidades principais e 0 caso contrário;

$t a_{c}$ : tempo de atendimento para o $\mathrm{CD} c$ atender o cliente $l$;

$f e_{c l}$ : frequência de entrega do $\mathrm{CD} c$ atendendo o cliente $l$;

$\operatorname{cod}_{c l}$ : consumo de $\mathrm{CO}_{2}$ na distribuição de uma moto do $\mathrm{CD} c$ ao cliente $l$;

cotcp: consumo de $\mathrm{CO}_{2}$ na transferência de uma moto feita da fábrica até o $\mathrm{CD} c$ passando pelo porto $p$;

catual: custo máximo aceitável, que representa o custo atual da operação logística;

cotimo: custo mínimo possível da solução ótima do modelo de otimização de custo operacional;

$p^{V}$ : peso do objetivo $v$ na função de valor da solução, $v \in \mathbf{V}$;

pco: peso do custo operacional na função de valor da solução.
Definindo novas variáveis auxiliares:

$y_{i}^{V}$ : variável auxiliar binária, com $i=1,2, \ldots, n \mathrm{e}$ $v \in \mathbf{V}$;

$z_{j}^{V}$ : variável auxiliar real, $\operatorname{com} i=1,2, \ldots, n+1 \mathrm{e}$ $v \in \mathbf{V}$.

nas quais:

- $v$ é o índice que caracteriza que a variável auxiliar em questão é relativa ao atributo que compõe a função de valor da solução; para o caso da aplicação, $\mathbf{V}$ tem 8 elementos e para cada um deles foram definidas $y_{1}, y_{2}, y_{3}, z_{1}, z_{2}, z_{3} e z_{4}$;

- $n$ é a quantidade de funções lineares que compõem a função de valor de cada elemento $v$.

Novas restrições:

$$
\begin{gathered}
z_{1}^{v} \leq y_{1}^{v}, v \in \boldsymbol{V} \\
z_{i+1}^{v} \leq y_{i}^{v}+y_{i+1}^{v}, i=1, \ldots, n-1 \text { e } v \in \boldsymbol{V}
\end{gathered}
$$

$$
z_{i+1}^{v} \leq y_{i}^{v}, i=n \text { e } v \in \boldsymbol{V}
$$

$$
\sum_{i=1}^{n} y_{i}^{v}=1, v \in \boldsymbol{V}
$$

$$
\sum_{i=1}^{n+1} z_{i}^{v}=1, v \in \boldsymbol{V}
$$

$$
\begin{aligned}
& \sum_{i=1}^{n+1} b_{i}^{v} z_{i}^{v}=\sum_{c \in C} z_{c}\left(1-e c_{c}\right) c a+ \\
& \sum_{c \in C}\left(1-z_{c}\right) e c_{c} c f, \text { para } v=\text { Investimento }
\end{aligned}
$$

$$
\begin{aligned}
& \sum_{i=1}^{n+1} b_{i}^{v} z_{i}^{v}=\sum_{l \in L} g_{l} \sum_{c \in C} q c l_{c l} t a_{c l} / \sum_{l \in L} d_{l} g_{l} \\
& \text { parav }=\text { Tempodeatendimento } C 1
\end{aligned}
$$

$\sum_{i=1}^{n+1} b_{i}^{v} z_{i}^{v}=\sum_{l \in L}\left(1-g_{l}\right) \sum_{c \in C} q c l_{c l} t a_{c l} / \sum_{l \in L} d_{l}\left(1-g_{l}\right)$,

para $v=$ Tempo de atendimento $C 2$

$$
\begin{aligned}
& \sum_{i=1}^{n+1} b_{i}^{v} z_{i}^{v}=\sum_{l \in L}\left(1-g_{l}\right) \sum_{c \in C} q c l_{c l} f e_{c l} / \sum_{l \in L} d_{l}\left(1-g_{l}\right), \\
& \text { para } v=\text { Frequência deentrega } C 2
\end{aligned}
$$

$$
\begin{aligned}
& \sum_{i=1}^{n+1} b_{i}^{v} z_{i}^{v}=\left(\begin{array}{l}
\sum_{c \in \boldsymbol{C}} \sum_{p \in \boldsymbol{P}} q p c_{p c} \cot t_{c p}+ \\
\sum_{c \in \boldsymbol{C} l \in \boldsymbol{L}} \sum_{l \in l} q c l_{c l} \operatorname{cod}_{c l}
\end{array}\right) / \\
& \sum_{l \in L} d_{l}, \text { para } v=\text { EmissãoCO }^{2} \\
& \sum_{i=1}^{n+1} b_{i}^{v} z_{i}^{v}=\sum_{c \in C} z_{c}\left(1-e c_{c}\right) \\
& \text { para } v=\text { Publicidade positiva }
\end{aligned}
$$




$$
\begin{gathered}
\sum_{i=1}^{n+1} b_{i}^{v} z_{i}^{v}=\sum_{c \in C}\left(1-z_{c}\right) e c_{c}, \\
\text { para } v=\text { Publicidadenegativa } \\
\sum_{i=1}^{n+1} b_{i}^{v} z_{i}^{v}=\sum_{c \in C} z_{c} \\
\text { para } v=\text { Complexidade } \\
\sum_{c \in \boldsymbol{C}} \sum_{p \in \boldsymbol{P}} q p c_{p c}\left(c p_{p}+c p c_{p c}\right)+ \\
\sum_{c \in \boldsymbol{C} l \in \boldsymbol{L}} \sum_{c c l} q c l_{c l} \leq \text { catual }
\end{gathered}
$$

As Restrições 11 a 15 estruturam as funções de valor de forma a representa-las, para cada um dos oito objetivos fundamentais acrescidos no segundo modelo, como funções lineares por partes. Já as Restrições 16 a 23 compatibilizam o valor da função modelada por partes ao cálculo de cada um dos indicadores que representam os objetivos. Para mais detalhes sobre modelagens de funções lineares por partes, ver Winston \& Goldberg (2003).

A Restrição 24 limita o custo operacional ao custo atual da operação. Essa restrição foi necessária porque a função de valor do custo operacional foi modelada linearmente.

A nova função objetivo fica:

Maximizar:

$$
\begin{aligned}
& \left(\sum_{v \in \boldsymbol{V}} \sum_{i=1}^{n+1} b_{i}^{v} z_{i}^{v} p^{v}+\right. \\
& \left.\left(\begin{array}{l}
\text { catual }-\sum_{c \in \boldsymbol{C}} \sum_{p \in \boldsymbol{P}} q p c_{p c}\left(c p_{p}+c p c_{p c}+c v_{c}\right)- \\
\sum_{c \in C l \in \boldsymbol{L}} \sum_{c l} q c l_{c l}
\end{array}\right) \cdot \frac{p c o}{(\text { catual }- \text { cotimo })}\right) \\
& \left(\sum_{v \in \boldsymbol{V}} p^{v}+p c o\right)
\end{aligned}
$$

A Função 25 é composta por dois elementos, sendo que o primeiro representa a somatória ponderada das funções de valor modeladas como funções lineares por partes, enquanto a segunda parte representa a função de valor do custo operacional, que foi modelada como uma função linear.

\section{Aplicação do modelo}

Anova realidade do mercado nos últimos anos apontou para a oportunidade de redefinirem-se as localizações dos centros de distribuição na região do NE, uma vez que foi a região que apresentou maior crescimento percentual e vem se tornando uma importante região de demanda para a empresa.

Foi feita uma aplicação do modelo de forma a considerar o planejamento logístico à Região NE, para o qual foram selecionadas como pontos de demanda as 40 cidades da Região NE com maior população. O volume de vendas real de 2012 foi utilizado como demanda, apurada no nível mesorregião do IBGE e distribuída proporcionalmente à venda das cidades selecionadas em cada mesorregião.

Como candidatos a centros de distribuição foram consideradas as 15 mais populosas cidades da Região NE além de Belém, localizada na Região Norte, que foi adicionada às cidades candidatas em virtude de ter o porto com maior movimentação de carga vinda da fábrica de Manaus.

O modelo de otimização foi carregado com os parâmetros definidos para obtenção da solução ótima (SolOtima), de forma que foi possível obter o valor de cada um dos objetivos referentes à solução, bem como o valor total da solução. O problema com um total de 780 variáveis e 187 restrições foi rodado em 1,9 segundos, com a obtenção de uma função objetivo que representa o valor da solução de 79,6.

Com o objetivo de levantar soluções alterativas para que essas fossem avaliadas e comparadas com a solução ótima, o modelo foi rodado outras três vezes com alterações de alguns parâmetros.

A primeira rodada foi feita com os pesos de todos os objetivos, além do custo operacional, iguais a zero. Esse processo foi feito para que uma solução de menor custo operacional (SolMinCusto) que atendesse todos os limites aceitáveis dos demais objetivos fosse obtida.

$\mathrm{O}$ mesmo processo de zerar o peso de certos objetivos foi feito na segunda rodada, porém com os pesos de todos os objetivos que não faziam parte do nível de serviço. Esse exercício gerou uma solução de melhor nível de serviço (SolNS) que atendesse todos os limites aceitáveis dos demais objetivos.

Para o último modelo rodado, todos os centros de distribuição atuais foram forçados e a quantidade máxima de centros de distribuição permitida pelo modelo foi igualada à quantidade de CDs atualmente ativos. Esse processo propôs uma solução de operação ótima que considera apenas a estrutura de CDs atual (SolAtual).

a. Resultados

Alguns detalhes das soluções obtidas foram omitidos para garantir o sigilo de informações estratégicas da empresa.

As quatro soluções obtidas foram analisadas em termos dos valores de cada atributo, o que comprovou que a SolOtima obteve um resultado significativamente melhor que as demais, com valor de 79,6, seguida da solução SolMinCusto, com valor de 70,5. A solução SolNS teve o terceiro melhor desempenho, com valor igual a 67,5 enquanto a solução SolAtual obteve o pior desempenho, com valor em 57,2, como pode ser visto na Tabela 2.

A solução SolOtima, apesar de não ter apresentado os maiores valores para a maioria dos atributos, tendo o maior valor apenas para os atributos Emissão de $\mathrm{CO}_{2}$ e Publicidade negativa, obteve o maior valor na função objetivo, com um aumento de $39 \%$ em relação à configuração atual, uma vez que conseguiu equilibrar todos os atributos, não tendo o pior desempenho em nenhum dos atributos. 
A solução SolMinCusto, como esperado, obteve o maior valor referente ao custo operacional, próximo a 100. Por outro lado, com uma redução de apenas $0,07 \%$ no custo operacional, essa solução teve piora significativa, sobretudo no atributo Complexidade, por ter aumentado a quantidade de CDs de cinco para nove. Além disso, essa solução propôs o fechamento de um $\mathrm{CD}$ atual e a abertura de outros cinco, o que também causou uma piora nos atributos Investimento e Publicidade negativa.

A solução SolNS obteve os maiores valores para os três atributos do grupo de nível de serviço e da Publicidade positiva. Por outro lado, a solução obteve o pior desempenho nos atributos Custo operacional, Complexidade, Investimento e Publicidade negativa. Essa solução propôs a abertura de seis novos CDs e o fechamento de dois CDs atuais, chegando a um total de nove CDs ativos.

A solução SolAtual apresentou o maior valor para os atributos que refletiam a existência dos cinco CDs atuais, com baixa complexidade, não necessitando investimento e sem publicidade negativa. Porém se mostrou com baixo desempenho nos atributos relacionados ao nível de serviço, na Emissão de $\mathrm{CO}_{2}$ e Publicidade positiva.

A comparação entre as soluções SolOtima e SolAtual permite identificar os benefícios que seriam obtidos com a adequação da rede logística da empresa. A redução de custo entre os dois cenários não foi tão significativa $(0,6 \%)$, porém a abertura de dois novos CDs, que implica também em um pequeno aumento na complexidade da operação, aumentando a quantidade de CDs de cinco para sete, permitirá também o aumento no valor de quase todos os demais atributos, com melhoria do nível de serviço, redução na Emissão de $\mathrm{CO}_{2}$ e melhoria na Publicidade positiva. A diferença entre os esses atributos para as duas soluções pode ser visto na Tabela 3.

Uma análise de sensibilidade dos pesos de cada um dos atributos também foi aplicada, a fim de verificar a robustez da solução. Foram aplicadas simulações para gerar curvas que retratassem o impacto que a variação individual de cada atributo tem no valor final da solução. $\mathrm{Na}$ Figura 3 foram apresentadas essas curvas para os

Tabela 2. Valores de cada atributo para as soluções comparadas.

\begin{tabular}{cccccr}
\hline Atributo & Peso & SolOtima & SolMinCusto & SolNS & SolAtual \\
\hline Custo operacional & $\mathbf{1 0 0}$ & 98,4 & 98,9 & 83,9 & 93,3 \\
Lead time C1 (h) & $\mathbf{7 5}$ & 85,2 & 85,0 & 95,1 & 5,0 \\
Lead time C2 (h) & $\mathbf{3 5}$ & 36,4 & 36,2 & 44,4 & 34,0 \\
Freq. entrega C2 & $\mathbf{1 0}$ & 49,5 & 49,5 & 52,6 & 41,3 \\
Emissão CO & $\mathbf{8}$ & 50,4 & 50,0 & 46,5 & 45,3 \\
Complexidade & $\mathbf{2 0}$ & 60,0 & 0,0 & 0,0 & 90,0 \\
Investimento & $\mathbf{1 5}$ & 75,0 & 35,8 & 25,0 & 100,0 \\
Publicidade neg. & $\mathbf{1 5}$ & 100,0 & 30,0 & 10,0 & 100,0 \\
Publicidade pos. & $\mathbf{1 0}$ & 70,0 & 88,8 & 95,0 & 0,0 \\
Valor final & & $\mathbf{7 9 , 6}$ & $\mathbf{7 0 , 5}$ & $\mathbf{6 7 , 5}$ & $\mathbf{5 7 , 2}$ \\
\hline
\end{tabular}

Tabela 3. Comparação de atributos entre as soluções SolOtima e SolAtual.

\begin{tabular}{cccccc}
\hline & $\begin{array}{c}\text { Lead time } \mathbf{C 1} \\
\text { (h) }\end{array}$ & $\begin{array}{c}\text { Lead time C2 } \\
\text { (h) }\end{array}$ & $\begin{array}{c}\text { Frequência } \\
\text { de entrega } \\
\text { semanal }\end{array}$ & $\begin{array}{c}\text { Emissão CO } \\
\text { (kg/produto) }\end{array}$ & $\begin{array}{c}\text { Quantidade } \\
\text { de CDs }\end{array}$ \\
\hline $\begin{array}{c}\text { Diferença } \\
\text { (SolOtima - SolAtual) }\end{array}$ & $-2,5$ & $-0,5$ & 0,2 & $-0,5$ & 2,0 \\
\hline
\end{tabular}

Custo Operacional

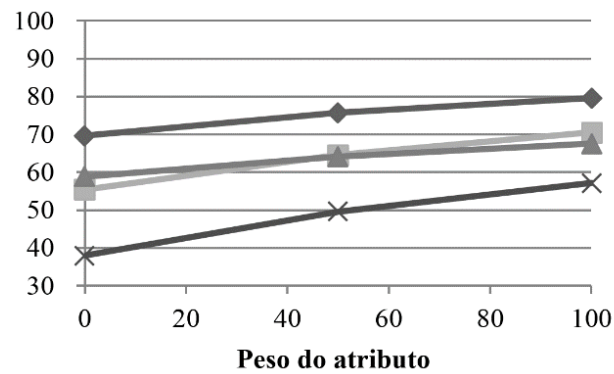

Lead Time C1

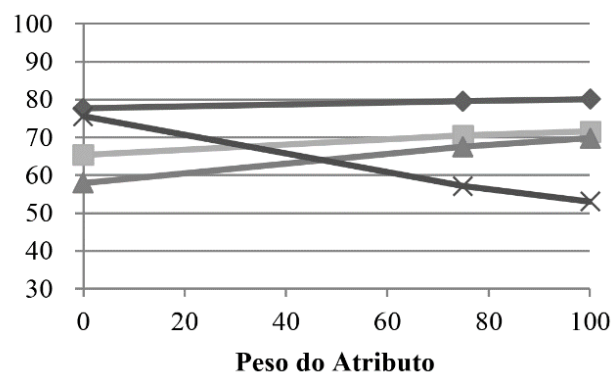

$\begin{array}{ll}\leftarrow \text { SolOtima } & - \text { SolMinCusto } \\ \leftarrow \text { SolNS } & \leftarrow \text { SolAtual }\end{array}$

Figura 3. Curvas de sensibilidade (Custo operacional e Lead time C1). 
atributos Custo operacional e Lead time das cidades principais $(\mathrm{C} 1)$, que mostram que a solução SolOtima se manteve como a solução de maior valor mesmo com a variação do peso dos atributos. As curvas dos demais atributos são apresentadas no Apêndice 1.

\section{Considerações finais}

O presente trabalho apresentou um modelo de matemático de otimização multicritério que considera importantes aspectos normalmente utilizados como critérios de decisão em grandes empresas, aplicado a um problema real de localização e alocação para uma indústria com unidade de produção localizada no Polo Industrial de Manaus. O problema foi representado por meio de um modelo de programação inteira mista com uma função objetivo composta por múltiplos critérios, entre eles custo operacional, nível de serviço dados em lead time e frequência de entrega, efeitos que impactam a sociedade, como emissão de $\mathrm{CO}_{2}$ e quantidade de unidades abertas e fechadas e efeitos que impactam a operação, como quantidade de CDs ativos.

O exercício permitiu a obtenção de uma solução que representa um aumento de pelo menos 39\% no valor da solução percebido pela empresa em relação à configuração atual, com redução do custo operacional ótimo, além de melhoria do nível de serviço prestado aos clientes em relação à operação atual. Também foram geradas soluções alternativas que permitiram identificar outras configurações possíveis à cadeia de distribuição, com as quais foram feitas análises de sensibilidade dos pesos de cada atributo.

Foi possível identificar que existe potencial de redução do custo operacional, melhoria no nível de serviço e redução da emissão de $\mathrm{CO}_{2}$ na operação com baixa quantidade de investimento, sendo necessária a abertura de dois novos CDs na região. Além disso, foi possível verificar que a solução de maior valor é robusta, mantendo-se a melhor solução mesmo com a variação de cada um dos atributos que fazem parte da função de valor.

Outros aspectos práticos que podem ser abordados em conjunto com os aspectos considerados no modelo, com o fim prover um suporte melhor à tomada de decisão, são:

- Consideração de incertezas no modelo (demanda, custos, capacidade);

- Consideração de elementos de risco para a função de valor;

- Localização de locais de produção;

- Múltiplos horizontes de planejamento;

- Outros métodos de criação de soluções alternativas, capazes de identificar outras configurações muito diferentes e com alto valor.
Os aspectos citados, entre outros, trazem mais complexidade ao exercício da modelagem matemática e representam desafios à aplicação de modelos de decisão realistas que consideram aspectos práticos do planejamento logístico.

A partir de uma análise na literatura científica foi possível identificar uma lacuna nos modelos de localização no que diz respeito a otimização multicritério. Uma vez que os aspectos considerados na função objetivo do modelo são em geral considerados à parte dos modelos de otimização, o ganho da abordagem de otimização multicritério nesse processo é claro.

\section{Agradecimentos}

Os autores agradecem o apoio à pesquisa pelo Conselho Nacional de Desenvolvimento Científico e Tecnológico, CNPq, pela bolsa de apoio a esta pesquisa.

\section{Referências}

Aardal, K., van den Berg, P. L., Gijswijt, D., \& Li, S. (2015). Approximation algorithms for hard capacitated k-facility location problems. European Journal of Operational Research, 242(2), 358-368. http://dx.doi.org/10.1016/j. ejor.2014.10.011.

Altiparmak, F., Gen, M., Lin, L., Paksoy, T. (2006). A genetic algorithm approach for multi-objective optimization of supply chain networks. Computers \& Industrial Engineering, 51(1), 196-215. http://dx.doi. org/10.1016/j.cie.2006.07.011.

Amaral, M., Almeida, M. S., \& Morabito, R. (2012). Um modelo de fluxos e localização de terminais intermodais para escoamento da soja brasileira destinada à exportação. Gestão \& Produção, 19(2), 717-732. http://dx.doi. org/10.1590/S0104-530X2012000400005.

Brandeau, M., \& Chiu, S. (1989). An overview of representative problems in location research. Management Science, 35(6), 645-674. http://dx.doi.org/10.1287/mnsc.35.6.645.

Current, J., Daskin, M., \& Schilling, D. (2002). Discrete network location models. In Z. Drezner \& H. Hamacher (Eds.), Facility location: applications and methods (pp. 81-118). Berlin: Springer-Verlag.

Daskin, M. M. S. (1995). Network and discrete location: models, algorithms, and applications (pp. 81-118). Berlin: John Wiley \& Sons.

Drezner, Z. (2004) Facility location: applications and theory (2nd ed). Springer.

Farahani, R. Z., \& Asgari, N. (2007). Combination of MCDM and covering techniques in a hierarchical model for facility location: A case study. European Journal of Operational Research, 176(3), 1839-1858. http:// dx.doi.org/10.1016/j.ejor.2005.10.039.

Farahani, R. Z., Hekmatfar, M., Fahimnia, B., \& Kazemzadeh, N. (2014). Hierarchical facility location problem: models, classifications, techniques, and applications. Computers 
\& Industrial Engineering, 68, 104-117. http://dx.doi. org/10.1016/j.cie.2013.12.005.

Farahani, R. Z., SteadieSeifi, M., \& Asgari, N. (2010). Multiple criteria facility location problems: a survey. Applied Mathematical Modelling, 34(7), 1689-1709. http://dx.doi.org/10.1016/j.apm.2009.10.005.

Franco, L., \& Montibeller, G. (2010). Problem structuring for multi-criteria decision analysis interventions. In J. J. Cochran (Eds.), Wiley Encyclopedia of Operations Research and Management Science. Chichester: John Wiley \& Sons. http://dx.doi.org/10.1002/9780470400531. eorms0683.

Goodwin, P., \& Wright, G. (2009). Decision analysis for management judgment (4th ed.). New York: John Wiley \& Sons.

Guazzelli, C. S., \& Cunha, C. B. (2014). Modelagem matemática para o problema de localização de centros de distribuição de empresa de alimentos. Revista Transportes, 22(3), 86-102. http://dx.doi.org/10.14295/ transportes.v22i3.767.

Guillén, G., Mele, F., Bagajewicz, M., Espuña, A., \& Puigjaner, L. (2005). Multiobjective supply chain design under uncertainty. Chemical Engineering Science, 60(6), 1535-1553. http://dx.doi.org/10.1016/j.ces.2004.10.023.

Harris, I., Mumford, C. L., \& Naim, M. M. (2014). A hybrid multi-objective approach to capacitated facility location with flexible store allocation for green logistics modeling. Transportation Research Part E, Logistics and Transportation Review, 66, 1-22. http://dx.doi. org/10.1016/j.tre.2014.01.010.

Hinojosa, Y., Kalcsics, J., Nickel, S., Puerto, J., \& Velten, S. (2008). Dynamic supply chain design with inventory. Computers \& Operations Research, 35(2), 373-391. http://dx.doi.org/10.1016/j.cor.2006.03.017.

Keeney, R. L., \& Gregory, R. S. (2005). Selecting attributes to measure the achievement of objectives. Operations Research, 53(1), 1-11. http://dx.doi.org/10.1287/ opre.1040.0158.

Latha Shankar, B., Basavarajappa, S., Chen, J. C. H., \& Kadadevaramath, R. S. (2013). Location and allocation decisions for multi-echelon supply chain network - A multi-objective evolutionary approach. Expert Systems with Applications, 40(2), 551-562. http://dx.doi. org/10.1016/j.eswa.2012.07.065.

Lee, D. H., \& Dong, M. (2008). A heuristic approach to logistics network design for end-of-lease computer products recovery. Transportation Research Part E, Logistics and Transportation Review, 44(3), 455-474. http://dx.doi.org/10.1016/j.tre.2006.11.003.

Mapa, S. M. S., \& Lima, R. D. S. (2012). Uso combinado de sistemas de informações geográficas para transportes e programação linear inteira mista em problemas de localização de instalações. Gestão \& Produção, 19(1), 119-136. http://dx.doi.org/10.1590/S0104$530 \times 2012000100009$.
Medeiros, R. L., Santos, J. T. A. N., Kuwahara, N., \& Moita, M. H. V. (2015). Cenários logísticos alternativos para a cabotagem do estado do Amazonas utilizando simulação computacional. JTL, 9(1), 60-64. http:// dx.doi.org/10.1590/2238-1031.jtl.v9n1a12.

Melo, M., Nickel, S., \& Saldanha-Da-Gama, F. (2009). Facility location and supply chain management-A review. European Journal of Operational Research, 196(2), 401-412. http://dx.doi.org/10.1016/j.ejor.2008.05.007.

Mirchandani, P., \& Francis, R. (1990). Discrete location theory. New York: Wiley.

Montibeller, G., \& Yoshizaki, H. (2011). A framework for locating logistic facilities with multi-criteria decision analysis. In R. H. C. Takahashi, K. Deb, E. F. Wanner, \& S. Greco (Eds.), Evolutionary multi-criterion optimization (Vol. 6576). Berlin: Springer. http://dx.doi. org/10.1007/978-3-642-19893-9_35.

Olivares-Benitez, E., Ríos-Mercado, R. Z., \& GonzálezVelarde, J. L. (2013). A metaheuristic algorithm to solve the selection of transportation channels in supply chain design. International Journal of Production Economics, 145(1), 161-172. http://dx.doi.org/10.1016/j. ijpe.2013.01.017.

Ozgen, D., \& Gulsun, B. (2014). Combining possibilistic linear programming and fuzzy AHP for solving the multi-objective capacitated multi-facility location problem. Information Sciences, 268, 185-201. http:// dx.doi.org/10.1016/j.ins.2014.01.024.

Pasandideh, S. H. R., Niaki, S. T. A., \& Asadi, K. (2014). Bi-objective optimization of a multi-product multi-period three-echelon supply chain problem under uncertain environments: NSGA-II and NRGA. Information Sciences, 292, 57-74. http://dx.doi.org/10.1016/j.ins.2014.08.068.

Passos, A. C., \& Souza, R. C. (2013). Defining a quality index for electric power utilities using multiple criteria decision support and time series analysis. Gestão \& Produção, 20(1), 1-12. http://dx.doi.org/10.1590/ S0104-530X2013000100001.

Pati, R., Vrat, P., \& Kumar, P. (2008). A goal programming model for paper recycling system. Omega, 36(3), 405417. http://dx.doi.org/10.1016/j.omega.2006.04.014.

Rahmaniani, R., \& Ghaderi, A. (2015). An algorithm with different exploration mechanisms: Experimental results to capacitated facility location/network design problem. Expert Systems with Applications, 42(7), 3790-3800. http://dx.doi.org/10.1016/j.eswa.2014.12.051.

ReVelle, C. S., \& Eiselt, H. A. (2005). Location analysis: a synthesis and survey. European Journal of Operational Research, 165(1), 1-19. http://dx.doi.org/10.1016/j. ejor.2003.11.032.

ReVelle, C. S., Eiselt, H. A., \& Daskin, M. S. (2008). A bibliography for some fundamental problem categories in discrete location science. European Journal of Operational Research, 184(3), 817-848. http://dx.doi. org/10.1016/j.ejor.2006.12.044. 
Sridharan, R. (1995). The capacitated plant location problem. Winston, W., \& Goldberg, J. (2003). Integer Programming. European Journal of Operational Research, 87(2), 203- O Operations research: applications and algorithms (4th 213. http://dx.doi.org/10.1016/0377-2217(95)00042-O. ed). Thomson/Brooks/Cole. 


\section{Apêndice 1.}

\section{Funções de valor}

São apresentadas as funções de valor de cada um dos atributos fundamentais levantados na análise com representantes da empresa e utilizados no modelo multicritério. No eixo horizontal dos gráficos são apresentadas as faixas possíveis e aceitáveis para cada atributo. Por questões de sigilo de informações estratégicas, os valores de custo operacional foram multiplicados por um fator.
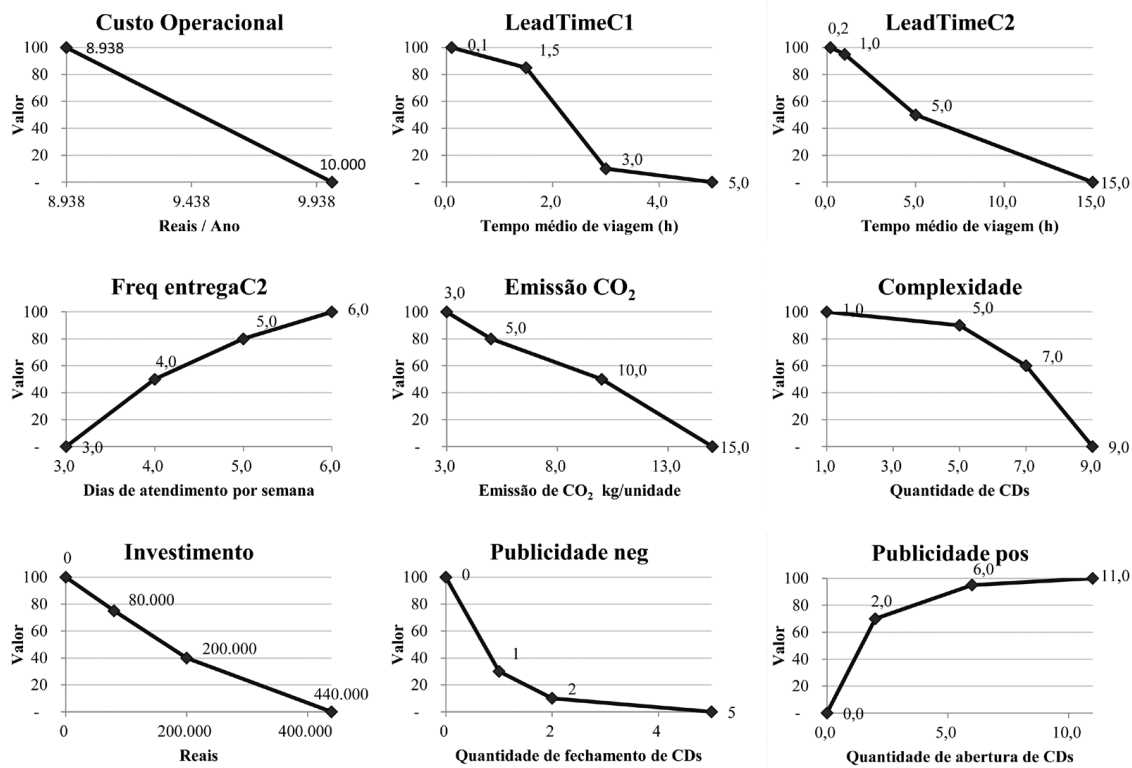

\section{Análise de sensibilidade}

Para cada um dos atributos, foram aplicadas simulações de sensibilidade, com o objetivo de verificar a robustez das soluções propostas. São apresentadas curvas que retrataram o impacto que a variação individual de cada atributo tem no valor final da solução.
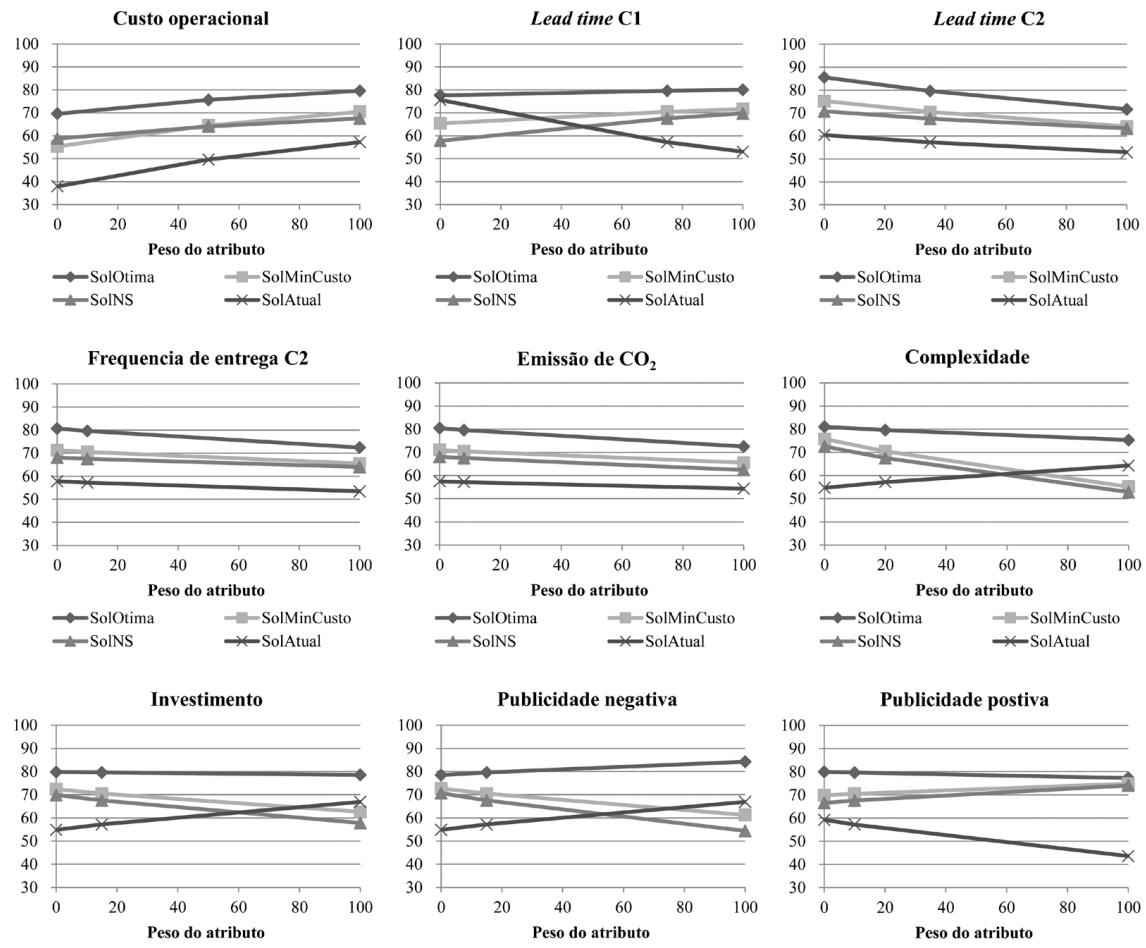

$\neg$ SolOtima $\quad-$-SolMinCusto $\underset{\sim}{\rightarrow}$ SolNs $\quad \approx$ SolAtual

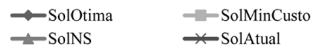

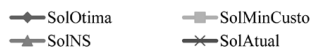

